\title{
STRUCTURE AND CALIBRATION OF CONSTITUTIVE EQUATIONS FOR GRANULAR SOILS
}

\author{
ANDRZEJ SAWICKI, JUSTYNA SŁAWIŃSKA, \\ JACEK MIERCZYŃSKI \\ Institute of Hydro-Engineering IBW PAN, ul. Kościerska 7, 80-328 Gdańsk, Poland, \\ e-mail: as@ibwpan.gda.pl; stynaju@ibwpan.gda.pl; mier@ibwpan.gda.pl
}

\begin{abstract}
The form of incremental constitutive equations for granular soils is discussed for the triaxial configuration. The classical elasto-plastic approach and the semi-empirical model are discussed on the basis of constitutive relations determined directly from experimental data. First, the general structure of elasto-plastic constitutive equations is presented. Then, the structure of semiempirical constitutive equations is described, and a method of calibrating the model is presented. This calibration method is based on a single experiment, performed in the triaxial apparatus, which also involves a partial verification of the model, on an atypical stress path. The model is shown to give reasonable predictions. An important feature of the semi-empirical incremental model is the definition of loading and unloading, which is different from that assumed in elasto-plasticity. This definition distinguishes between spherical and deviatoric loading/unloading. The definition of deviatoric loading/unloading has been subject to some criticism. It was therefore discussed and clarified in this paper on the basis of the experiment presented.
\end{abstract}

Key words: granular soil, constitutive equations, triaxial conditions, pre-failure deformations

\section{INTRODUCTION}

The aim of this paper is to discuss the structure of incremental constitutive equations for granular soils. The classical elasto-plastic approach and the semi-empirical model are discussed on the basis of constitutive relations determined directly from experimental data. First, the general structure of elasto-plastic constitutive equations is presented. Then, the structure of semi-empirical constitutive equations is described, and a method of calibrating the model is presented. This calibration method is based on a single experiment, performed in the triaxial apparatus, which also involves a partial verification of the model, on an atypical stress path. The model is shown to give reasonable predictions. An important feature of the semi-empirical incremental model is the definition of loading and unloading, which is different from that assumed in elastoplasticity. This definition distinguishes between spherical and deviatoric loading/unloading. The definition of deviatoric loading/unloading has been subject to some criticism. It was therefore discussed and clarified in this paper on the basis of the experiment presented.

\section{GENERAL FORM OF CONSTITUTIVE EQUATIONS}

The problem of constitutive equations for granular soils will be presented for the triaxial configuration, as theoretical models of soils are usually calibrated in such conditions. The pre-failure behaviour of soils is non-linear, so it is convenient to define constitutive equations in the incremental form. It is also convenient to decompose the stress and strain tensors into their spherical and deviatoric parts. The general form of constitutive equations for the triaxial configuration can be presented as follows

$$
\begin{aligned}
& d \varepsilon_{v}=\frac{\partial \varepsilon_{v}}{\partial p^{\prime}} d p^{\prime}+\frac{\partial \varepsilon_{v}}{\partial q} d q=M d p^{\prime}+N d q, \\
& d \varepsilon_{q}=\frac{\partial \varepsilon_{q}}{\partial p^{\prime}} d p^{\prime}+\frac{\partial \varepsilon_{q}}{\partial q} d q=P d p^{\prime}+Q d q
\end{aligned}
$$

where

$$
p^{\prime}=\left(\sigma_{1}^{\prime}+2 \sigma_{3}^{\prime}\right) / 3-\text { mean effective stress; }
$$
$q=\sigma_{1}^{\prime}-\sigma_{3}^{\prime}-$ deviatoric stress; $\varepsilon_{v}=\varepsilon_{1}+2 \varepsilon_{3}-$ volumetric strain; $\varepsilon_{q}=\frac{2}{3}\left(\varepsilon_{1}-\varepsilon_{3}\right)-$ deviatoric strain. Sub- 
script " 1 " corresponds to the vertical direction, and " 3 " to the radial direction. The soil mechanics sign convention is applied, where a positive sign denotes compression. $M, N, P$ and $Q$ are functions that generally depend on stress and strain states, and may also depend on some other factors, such as, for example, the initial state of soil.

Equations (1) and (2) are expressed in terms of total strain increments, and so far no distinction is made between plastic and elastic strains. An interesting feature of equations (1) and (2) is that they include functions $N$ and $P$, which are usually equal to zero for most engineering materials. The function $N$ describes the effect of dilation (volumetric changes due to shearing), which is characteristic of granular soils and can be neglected in other materials. The function $P$ describes the effect of anisotropy, which is usually neglected in most soil models. This is the transverse isotropy, induced by gravity, which is clearly observed in experimental results, cf. Sawicki and Świdziński [8]. In some publications, and even textbooks, see Atkinson [1], a symmetry of equations (1) and (2), i.e., $N=P$, is suggested, which is not correct, as the effect of dilation cannot be identified with the anisotropic structure of soil samples. These effects represent two different physical phenomena.

The basic problem of soil mechanics is to determine the constitutive functions $M, N, P$ and $Q$. Having established these functions, one can determine the prefailure deformations of soil for arbitrary stress paths. However, it is not an easy task for many reasons, and that is why so many models of soils have been proposed. The most stable point of soil mechanics is the Coulomb-Mohr yield condition, established already in the 18th century. It is a limit state condition that can be checked, with different accuracies, in almost any geotechnical laboratory. In this paper, the limit state of soil means the same as the critical or steady states. Recall that all these definitions denote physically the same phenomenon, namely an unconfined flow of soil under constant stresses and constant volume.

Sometimes it is convenient to present equations (1) and (2) in an alternative form, introducing $p^{\prime}$ and $\eta=$ $q / p^{\prime}$ as independent stress variables. In such a case, the incremental equations take the following form

$$
\begin{aligned}
& d \varepsilon_{v}=\frac{\partial \varepsilon_{v}}{\partial p^{\prime}} d p^{\prime}+\frac{\partial \varepsilon_{v}}{\partial \eta} d \eta=M^{\prime} d p^{\prime}+N^{\prime} d \eta, \\
& d \varepsilon_{q}=\frac{\partial \varepsilon_{q}}{\partial p^{\prime}} d p^{\prime}+\frac{\partial \varepsilon_{q}}{\partial \eta} d \eta=P^{\prime} d p^{\prime}+Q^{\prime} d \eta,
\end{aligned}
$$

where

$$
d \eta=\frac{1}{p^{\prime}}\left(d q-\eta d p^{\prime}\right) .
$$

It can be easily checked that the following relations hold

$$
\begin{array}{cc}
M^{\prime}=M+\eta N ; & N^{\prime}=p^{\prime} N ; \\
P^{\prime}=P+\eta Q ; & Q^{\prime}=p^{\prime} Q .
\end{array}
$$

\section{INCREMENTAL EQUATIONS IN CLASSICAL ELASTO-PLASTICITY}

As already mentioned, the problem of determining the constitutive functions $M, N, P$ and $Q$ is a key issue in soil mechanics. Determination of these functions is sometimes designated as the calibration of a constitutive model. Such calibration is usually performed in the triaxial apparatus, which is the most common experimental device in geotechnical laboratories. In determining the constitutive functions, an important role is played by the definition of loading/unloading, which is not a trivial problem. Let us see what the problem of incremental equations looks like in classical elasto-plasticity, cf. Wood [11].

The basic assumption is the decomposition of the total strain tensor increment $d \boldsymbol{\varepsilon}$ into elastic $d \boldsymbol{\varepsilon}^{e}$ and plastic $d \boldsymbol{\varepsilon}^{p}$ parts, as follows

$$
d \boldsymbol{\varepsilon}=d \boldsymbol{\varepsilon}^{e}+d \boldsymbol{\varepsilon}^{p} .
$$

In a similar way, the constitutive functions appearing in equations (1) and (2) can be decomposed into elastic and plastic parts for example, $M=M^{e}$ $+M^{p}$, etc. Both parts of constitutive functions should be determined either from experiments or from a priori assumptions. For example, it can be assumed that the elastic part of soil deformations obeys Hooke's law, which is obviously a kind of first approximation. Elastic moduli, however, should be determined from experiments performed for a given sand. In order to determine plastic strain increments, a specific methodology has been developed since the 1950's. Unfortunately, this methodology has been copied from the plasticity of metals, and that is why it has led to the creation of a large number of soil models, that do not always satisfy the basic scientific criterion, i.e., conformity with experimental data, see Saada and Bianchini [5]. However, it is the most common methodology, already accepted in geotechnical engineering, so some attention should be devoted to this fundamental problem. In the elasto-plastic approach, two funda- 
mental functions are introduced. One is the yield function (yield locus) $f=0$, which states whether the current load increment denotes loading or unloading. The function $f$ depends on the current stress state and may also depend on some other independent variables, such as, for example, the current strain.

During loading, the current yield function expands, and the condition defining this process can be expressed as follows

$$
\frac{d f}{d \boldsymbol{\sigma}} d \boldsymbol{\sigma} \geq 0
$$

Inequality (8) means that the subsequent stress increment is directed outwards to the current yield surface or is tangent to it. It is assumed that during loading both elastic and plastic strains develop. During unloading, only elastic strains are recovered. This process can be represented by stress increments directed inwards to the current yield surface, which can be expressed as follows

$$
\frac{d f}{d \boldsymbol{\sigma}} d \boldsymbol{\sigma}<0 .
$$

The change in the size of the yield loci may be linked to plastic strain increments, which are defined by a hardening rule. Suppose, as it was assumed in the Cam-Clay model, that the yield surface is of an elliptic shape, the size of which is controlled by the parameter $p_{0}^{\prime}$. In this case, a hardening rule has the following general form, cf. Wood [11]

$$
d p_{0}^{\prime}=\frac{\partial p_{0}^{\prime}}{\partial \varepsilon_{v}^{p}} d \varepsilon_{v}^{p}+\frac{\partial p_{0}^{\prime}}{\partial \varepsilon_{q}^{p}} d \varepsilon_{q}^{p} .
$$

In order to determine the plastic strain increments, another function $g$, designated as the plastic potential, is introduced. The plastic strain increments can be determined from the following relations

$$
\begin{aligned}
& d \varepsilon_{v}^{p}=\lambda \frac{\partial g}{\partial p^{\prime}}, \\
& d \varepsilon_{q}^{p}=\lambda \frac{\partial g}{\partial q},
\end{aligned}
$$

where $\lambda$ is a scalar multiplier.

The above assumptions lead to the following form of incremental equations for the plastic strains

$$
\left\{\begin{array}{l}
d \varepsilon_{v}^{p} \\
d \varepsilon_{q}^{p}
\end{array}\right\}=\Pi\left[\begin{array}{ll}
\frac{\partial f}{\partial p^{\prime}} \frac{\partial g}{\partial p^{\prime}} & \frac{\partial f}{\partial q} \frac{\partial g}{\partial p^{\prime}} \\
\frac{\partial f}{\partial p^{\prime}} \frac{\partial g}{\partial q} & \frac{\partial f}{\partial q} \frac{\partial g}{\partial q}
\end{array}\right]\left\{\begin{array}{c}
d p^{\prime} \\
d q
\end{array}\right\},
$$

where

$$
\Pi=-\left\{\frac{\partial f}{\partial p_{0}^{\prime}}\left[\frac{\partial p_{0}^{\prime}}{\partial \varepsilon_{v}^{p}} \frac{\partial g}{\partial p^{\prime}}+\frac{\partial p_{0}^{\prime}}{\partial \varepsilon_{q}^{p}} \frac{\partial g}{\partial q}\right]\right\}^{-1} .
$$

Note that the form of equations (13) is the same as that of equations (1) and (2), where $M^{p}=\Pi \frac{\partial f}{\partial p^{\prime}} \frac{\partial g}{\partial p^{\prime}}$, etc. If the associated flow rule is assumed, i.e. when $f=g$, equations (9) become symmetric. Recall that the function $N$ describes the effect of dilation, whereas $P$ describes the effect of anisotropy. These effects are not combined and the situation when $N=P$ is rather unrealistic in practice. On the other hand, the effect of anisotropy, either inherent or stressinduced, has been ignored in the derivation of equations (13), but it is clearly displayed in the final equations. How are we to explain this apparent inconsistency? No comments on this problem could be found in the available literature.

The other important issue is the determination of the constitutive functions $f$ and $g$, not to mention a law describing the elastic response of soil. They play a key role in the development of elasto-plastic models of soils, so their relevance, as well as details regarding their form, development, etc., should be confirmed experimentally. Again, one can hardly find convincing empirical arguments in the available literature.

Another shortcoming of classical elasto-plastic models is that the initial state of soil, defined as either dilative or contractive, is not taken into account. These states significantly influence the volumetric changes in soil during shearing and, consequently, the respective constitutive equations, see Sawicki and Świdziński [8]. General elasto-plastic constitutive equations (9) do not take into account this important fact. The above arguments mean that the elasto-plastic models of soils should probably be subjected to major revisions that take into account both the theoretical structure of these models and their agreement with experimental data.

Some shortcomings of the elasto-plastic approach to modeling the behaviour of soils have been noted earlier, and a few alternative approaches, such as the endochronic theory (Valanis [10]), (Bazant and Krizek [2]) or hypoplasticity (Kolymbas [4]), have been proposed. At present, the endochronic theory is not widely used and seems to be of historical value. Hypoplasticity is applied by a rather limited number of researchers, mainly those having contacts with the Karlsruhe school of geomechanics, see Gudehus [3]. It is not the aim of this paper to review these ap- 
proaches, as the elasto-plastic paradigm still prevails in soil mechanics.

\section{EMPIRICAL PIECE-WISE LINEAR INCREMENTAL EQUATIONS}

However, because of some shortcomings and questions regarding elasto-plasticity, an alternative has subsequently been proposed in detail by Sawicki and Świdziński [8], [9]. Their approach is based on a straightforward determination of the constitutive functions $M, N, P$ and $Q$ from the stress-strain curves, obtained from experimental investigations in the triaxial apparatus. An almost natural way of determining these functions is to perform relatively simple experiments on dry or saturated, but fully drained, soil samples for the stress paths shown in Fig. 1. In this case, $p=p^{\prime}$, where $p$ is the total mean stress.

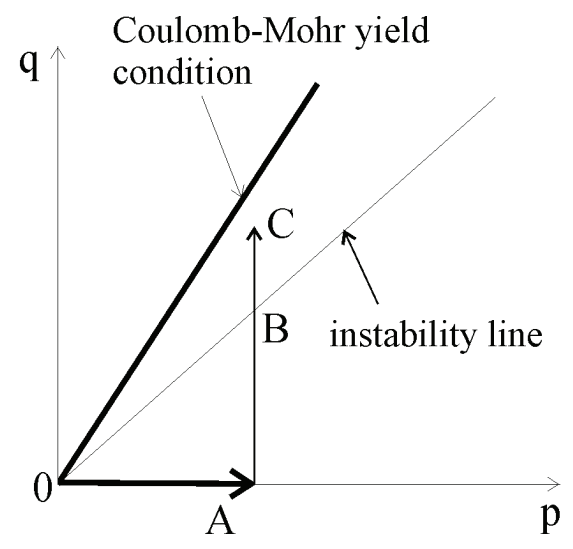

Fig. 1. Simple stress paths applied for the calibration of equations (1) and (2)

Path $0 \mathrm{~A}$, characterized by $d q=0$, corresponds to spherical loading. Such an experiment leads to the determination of the stress-strain curves, which have the following general form

$$
\begin{gathered}
\varepsilon_{v}=\varepsilon_{v}^{(p)}\left(p, I_{D}^{0}\right), \\
\varepsilon_{q}=\varepsilon_{q}^{(p)}\left(p, I_{D}^{0}\right),
\end{gathered}
$$

where $I_{D}^{0}$ denotes the initial density index of the soil sample, and the superscript ${ }^{(\mathrm{p})}$ means that these strains were caused by the mean stress $p=p^{\prime}$. Experimental results show that in this case $\varepsilon_{q} \neq 0$, which means that the soil samples display a kind of inherent anisotropy. It is a cross-isotropic behaviour, induced by gravity, which is usually ignored in most of the soil mechanics models. Differentiation of equations (14) and (15) with respect to $p$ leads to the determination of the constitutive functions $M$ and $P$. The same procedure can be applied for spherical unloading $(d p<0)$ and respective functions determined. For details, see Sawicki and Świdziński [8].

Another set of experiments deals with the stress path $\mathrm{ABC}$, which corresponds to pure shearing at a constant mean effective stress. It should be noted that such experiments are difficult to perform in a standard geotechnical laboratory for technical reasons. Most standard triaxial apparatuses are not capable of controlling the stress path $\mathrm{ABC}$, just offering "geotechnical stress paths", which are based on controlling the vertical stress and keeping the cell pressure constant. These experiments lead to the determination of respective stress-strain curves, namely

$$
\begin{aligned}
& \varepsilon_{v}=\varepsilon_{v}^{(q)}\left(p_{0}, q, I_{D}^{0}\right), \\
& \varepsilon_{q}=\varepsilon_{q}^{(q)}\left(p_{0}, q, I_{D}^{0}\right),
\end{aligned}
$$

where $p_{0}$ denotes the mean stress at point $\mathrm{A}$, which is constant along the path $\mathrm{ABC}$. The superscript (q) means that the strains (16) and (17) were caused by deviatoric strain. The experiments were performed for different values of $p_{0}=$ const, leading to different stress-strain curves for each value of $p_{0}$, which, in this case, plays a role of parameter. At first sight, such experimental data are difficult to comprehend, as one deals with a large number of curves, each corresponding to different values of $p_{0}$ and $I_{D}^{0}$. However, by trial and error, one can eventually put this abundant information in order. There are two important issues which should be taken into account.

The first is the initial state of soil, designated either as contractive or dilative. The experimental results show that the volumetric behaviour of soils strongly depends on this state. A soil that is initially contractive compacts as a result of shearing, whereas an initially dilative soil compacts first and then dilates (increases in volume because of shearing). The parameter $I_{D}^{0}$ is less important in this case. The key role is played by the position of the initial state of soil on the $p^{\prime}, e$ plane, where $e$ denotes the voids ratio. The $p^{\prime}$, e plane is divided into two parts, separated by the steady state line (SSL), which corresponds to the plastic flow of soil under constant volume and stresses. The region above this line corresponds to the contractive behaviour of soil, whereas the region below defines dilative behaviour. SSL should be determined experimentally, see Sawicki and Świdziński [8]. Therefore, the different forms of the strain-stress curves (16) should be determined for these different 
initial states. The forms of the stress-strain curves (17) are similar for both, initially contractive and dilative samples.

The second problem is how to present experimental results. The method of trial and error led to the following representation of these results

$$
\begin{aligned}
& \frac{\varepsilon_{v}^{(q)}}{\sqrt{p_{0}}}=F_{v}(\eta), \\
& \frac{\varepsilon_{q}^{(q)}}{\sqrt{p_{0}}}=F_{q}(\eta),
\end{aligned}
$$

where $\eta=q / p_{0}$ in this case. An advantage of such presentation is that different curves, corresponding to different values of $p_{0}$, form roughly a single common curve, which can be approximated by analytical formulae. Differentiation of these curves with respect to $\eta$ leads to the determination of functions $N$ and $Q$. Note that $p_{0}$ is treated as a parameter in this case. Examples of these functions are shown in Sawicki and Świdziński [8], [9]. The same procedure can be applied to deviatoric unloading, i.e., when $d q<0$.

Instability line plays a key role in mechanics of granular soils. The behaviour of initially dilative and contractive soils is different after approaching this line during shearing. Note that this line does not depend on the loading path. After approaching this line, the initially contractive sand still densifies but the initially dilative sand dilates. During the undrained shearing, the static liquefaction take place in the case of initially contractive soil. For details, see Sawicki and Świdziński [8], [9], Sawicki [6], [7].

\section{LOADING AND UNLOADING}

In the previous section, a natural definition of loading and unloading was introduced for the spheri- cal and deviatoric parts of the stress tensor separately. Recall that we have defined these processes as follows:

$d p^{\prime}>0$ - spherical loading,

$d p^{\prime}<0$ - spherical unloading,

$d q>0$ - deviatoric loading,

$d q<0$ - deviatoric unloading.

Consequently, the constitutive functions $M, N, P$ and $Q$ have different forms, depending on whether they correspond to loading or unloading. Let the superscript ()$^{l}$ denote loading, and ()$^{u}$ unloading. For example, $M^{l}$ is the function $M$ for loading, and $M^{u}$ is the function for unloading, etc. In order to illustrate this idea, let us consider successive stress increments shown in Fig. 2. The corresponding constitutive equations are shown in Table 1.

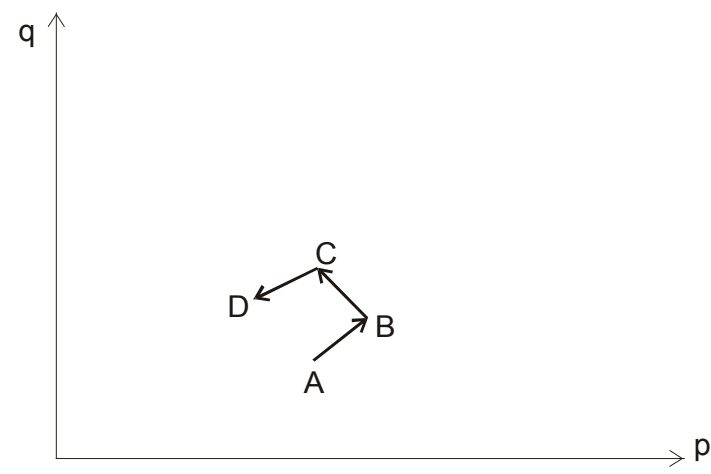

Fig. 2. Successive stress increments

The definition of loading and unloading introduced here is certainly different from that applied in elasto-plasticity and appears to be more natural and self-contained. Recall that the "elasto-plastic" definition of loading/unloading depends on the assumed shape of the current yield surface and therefore is subjective, since for different models the same stress increment may be considered as either loading or unloading - with corresponding consequences. Also recall that according to classical elasto-plasticity,

Table 1. Incremental equations for successive stress increments, shown in Fig. 2

\begin{tabular}{|l|l|l|}
\hline $\begin{array}{c}\text { Stress } \\
\text { increment }\end{array}$ & \multicolumn{1}{c|}{ Process } & \multicolumn{1}{c|}{$\begin{array}{c}\text { Equations for respective } \\
\text { strain increments }\end{array}$} \\
\hline AB & $\begin{array}{l}d p>0 \text { - spherical loading } \\
d q>0-\text { deviatoric loading }\end{array}$ & $\begin{array}{l}d \varepsilon_{v}=M^{l} d p+N^{l} d q \\
d \varepsilon_{q}=P^{l} d p+Q^{l} d q\end{array}$ \\
\hline BC & $\begin{array}{l}d p<0 \text { - spherical unloading } \\
d q>0-\text { deviatoric loading }\end{array}$ & $\begin{array}{l}d \varepsilon_{v}=M^{u} d p+N^{l} d q \\
d \varepsilon_{q}=P^{u} d p+Q^{l} d q\end{array}$ \\
\hline $\mathrm{CD}$ & $d p<0-$ spherical unloading & $d \varepsilon_{v}=M^{u} d p+N^{u} d q$ \\
& $d q<0-$ deviatoric unloading & $d \varepsilon_{q}=P^{u} d p+Q^{u} d q$ \\
\hline
\end{tabular}


plastic strains develop during loading. It cannot be accepted that according to one model, a given stress increment produces plastic strains, whereas according to another model the same increment means unloading, i.e., only recovered elastic strains. Also note that definitions of loading/unloading introduced in elastoplasticity and in the present model have different physical meaning. In elasto-plasticity, loading means the development of plastic strains, whereas in the current approach loading means just an increase in the respective part of the stress tensor (spherical and deviatoric) without any reference to strains.

The definition of loading and unloading discussed in this section is unique as shown in Fig. 3. In order to calculate strains that develop along an arbitrary loading path, one should integrate incremental equations (1) and (2) according to assumed rules, illustrated in Table 1. Recall that the stress increment AB, shown in Fig. 3, corresponds to unloading, both spherical and deviatoric, and each stress increment along this stress path can be defined precisely as spherical/deviatoric loading or unloading.

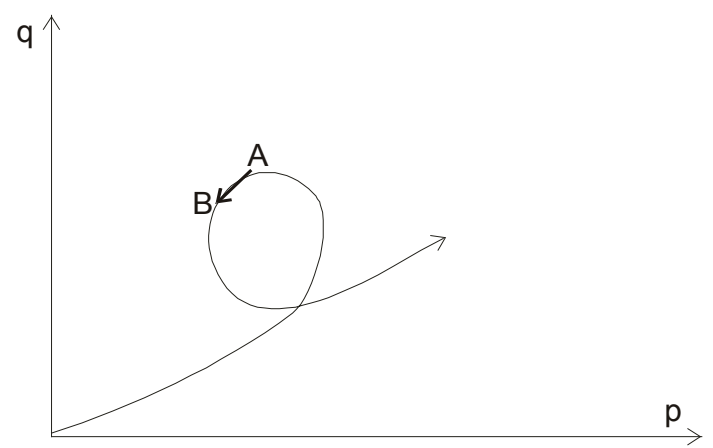

Fig. 3. Each stress increment along an arbitrary stress path is defined uniquely

\section{Alternative definition of deviatoric loading/unloading}

The following dimensionless stress variable has already been introduced: $\eta=q / p$. An alternative definition of deviatoric loading can be defined as $d \eta>0$, and deviatoric unloading as $d \eta<0$. This alternative definition sometimes coincides with the previous definition, introduced at the beginning of this section, but in certain cases these two definitions deal with different physical phenomena as an increase or a decrease in the corresponding strain, which will be shown later. Anticipating the experimental results, we can assume that the alternative definition of loading/unloading is physically more sound than the original proposition. In the case considered, the incremental equations (3) and (4) are valid.

\section{METHOD OF CALIBRATION}

Sawicki and Świdziński [8] have proposed a method of calibrating the constitutive equations that uses the stress-strain curves obtained from the experiments depicted in Fig. 1. The first set of experiments dealt with spherical loading and unloading along the stress path $0 \mathrm{~A} 0$. In this case, $d q=0, d \eta=0, \eta=0$. Experimental results can be presented in the form of equations (14) and (15). This experiment makes it possible to determine the functions $M$ and $P$ by differentiation of equations (14) and (15) with respect to $p^{\prime}$. For example, a good analytical approximation of experimental results is the following

$$
\begin{gathered}
\varepsilon_{v}^{\left(p^{\prime}\right)}=A_{v} \sqrt{p^{\prime}}, \\
\varepsilon_{q}^{\left(p^{\prime}\right)}=A_{q} \sqrt{p^{\prime}},
\end{gathered}
$$

where $A_{v}$ and $A_{q}$ depend on the initial void ratio. Note that the analytical approximations (20) and (21) are not unique, as one can find some other analytical formulae approximating the experimental data equally well. For example, the function

$$
\varepsilon_{v}^{\left(p^{\prime}\right)}=A_{v}^{\prime} \ln \left(1+p^{\prime}\right)
$$

is also acceptable. A practical problem is to find analytical approximations in the simplest possible form, just for practical reasons. Differentiation of the functions (20) and (21) with respect to $p^{\prime}$ leads to the following formulae for the constitutive functions $M$. and $P$, see equations (3) and (4). Note that at the stress path $0 \mathrm{~A}$, there is $\eta=0$, so in this case $M=M^{\prime}$ and $P=P^{\prime}$. The corresponding functions are the following

$$
M^{l}=\frac{A_{v}}{2 \sqrt{p^{\prime}}}, \quad P^{l}=\frac{A_{q}}{2 \sqrt{p^{\prime}}},
$$

where the superscript ${ }^{l}$ denotes that these functions correspond to spherical loading.

Consider now the process of deviatoric loading along the path $\mathrm{ABC}$ in Fig. 1, where $d p^{\prime}=0, d q>0$, $d \eta>0$. In the case of an initially contractive soil, the following functions approximate experimental data, see Sawicki and Świdziński [8]

$$
\begin{gathered}
\varepsilon_{v}^{(\eta)}=c_{1} \sqrt{p^{\prime}} \eta^{4} \\
\varepsilon_{q}^{(\eta)}=b_{1} \sqrt{p^{\prime}}\left[\exp \left(b_{2} \eta\right)-1\right],
\end{gathered}
$$

where $c_{1}, b_{1}, b_{2}$ are coefficients (numbers) typical of a given sand. Partial differentiation of these equations 
with respect to $\eta$, cf. equations (3) and (4), leads to the following formulae

$$
\begin{gathered}
N^{\prime}=4 c_{1} \sqrt{p^{\prime}} \eta^{3}=p^{\prime} N^{l}, \\
Q^{\prime}=b_{1} b_{2} \sqrt{p^{\prime}} \exp \left(b_{2} \eta\right)=p^{\prime} Q^{l} .
\end{gathered}
$$

A similar procedure can be applied for the processes of spherical and deviatoric unloading. Details are presented in Sawicki and Świdziński [8]. Bear in mind that the forms of analytical approximations of experimental data may differ from those presented in this section. They depend on specific empirical results and the researcher's inventiveness, cf. equations (20) and (22).

\section{SOME EXPERIMENTAL DATA}

In the present and following sections some new experimental data will be presented and analyzed according to the approach discussed in this paper. The experiments were performed in a GDS triaxial apparatus equipped with special gauges for the local measurement of both vertical and lateral strains. Quartz "Skarpa" sand was used in these experiments. The basic characteristics of this sand are the following: median size of grains $D_{50}=420 \mu \mathrm{m}$, uniformity coefficient $C_{u}=2.5$, specific gravity $G=2.65$, maximum and minimum void ratios $e_{\max }=0.677$ and $e_{\min }=$ 0.432 , angles of internal friction $\varphi=34^{\circ}$ and $41^{\circ}$ for loose and dense sand, respectively. Soil samples were prepared in a membrane-lined split moulder, either by moist tamping (loose, contractive sample) or by water pluviation (dense, dilative sample) methods. Figure 4 shows the stress paths applied during the experiments: $0 \mathrm{ABC}$ and then CBA0. Some comments on the successive sectors of these paths are provided in Table 2.

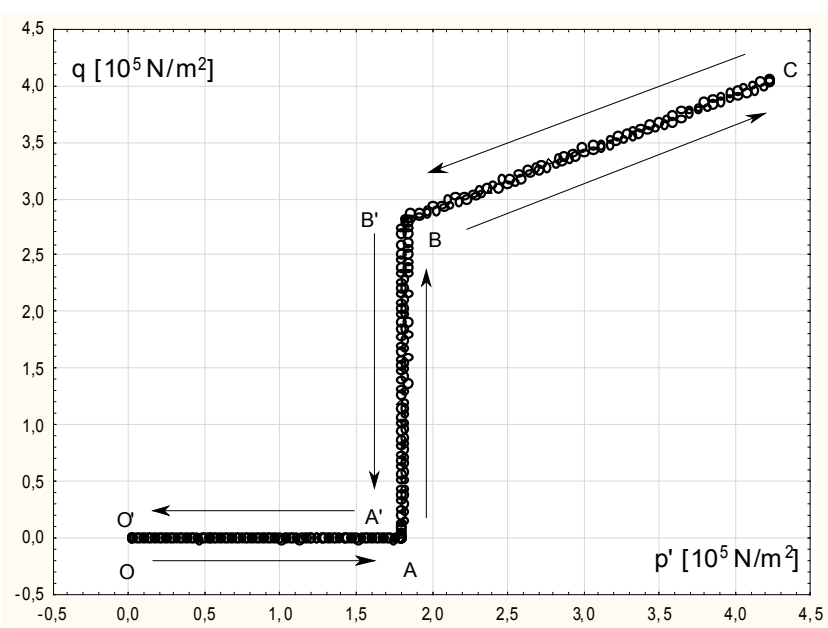

Fig. 4. The stress path applied in the experiments
Table 2. Interpretation of stress increments along the stress path shown in Fig. 4

\begin{tabular}{|c|c|l|}
\hline Sector & Stress increment & \multicolumn{1}{|c|}{ Comment } \\
\hline 0A & $d p^{\prime}>0, d q=0, d \eta=0$ & $\begin{array}{l}\text { pure spherical loading, } \\
\text { zero deviatoric stress }\end{array}$ \\
\hline $\mathrm{AB}$ & $d p^{\prime}=0, d q>0, d \eta>0$ & $\begin{array}{l}\text { pure deviatoric loading, } \\
\text { constant mean stress }\end{array}$ \\
\hline $\mathrm{BC}$ & $d p^{\prime}>0, d q>0, d \eta<0$ & $\begin{array}{l}\text { spherical loading, } \\
\text { deviatoric loading or unloading? }\end{array}$ \\
\hline $\mathrm{CB}$ & $d p^{\prime}<0, d q<0, d \eta>0$ & $\begin{array}{l}\text { spherical unloading, } \\
\text { deviatoric loading or unloading? }\end{array}$ \\
\hline $\mathrm{BA}$ & $d p^{\prime}=0, d q<0, d \eta<0$ & $\begin{array}{l}\text { pure deviatoric unloading, } \\
\text { constant mean stress }\end{array}$ \\
\hline $\mathrm{A} 0$ & $d p^{\prime}<0, d q=0, d \eta=0$ & $\begin{array}{l}\text { pure spherical unloading, } \\
\text { zero deviatoric stress }\end{array}$ \\
\hline
\end{tabular}

Careful analysis of Table 2 is necessary to define proper loading/unloading criteria, as it is important for the calibration of incremental equations and their application to study the pre-failure behaviour of sand. The definition of spherical loading/unloading is unproblematic, but some questions arise regarding the definition of deviatoric loading/unloading. The question is whether to choose the sign of $d q$ or the sign of $d \eta$ to define these processes. The method of calibration presented in the previous sections is does not raise doubts, as along the paths $\mathrm{AB}$ and $\mathrm{BA}$ both criteria coincide ( $d q$ and $d \eta$ have the same sign), and then the corresponding constitutive functions $M, N, P$ and $Q$ are properly determined. Note that the signs of these stress increments are different along the paths $\mathrm{BC}$ and $\mathrm{CB}$. In order to solve this dilemma, it is necessary to analyse strains that develop along these stress paths. Figure 5 shows the strain path corresponding to the stress path from Fig. 4 for an initially

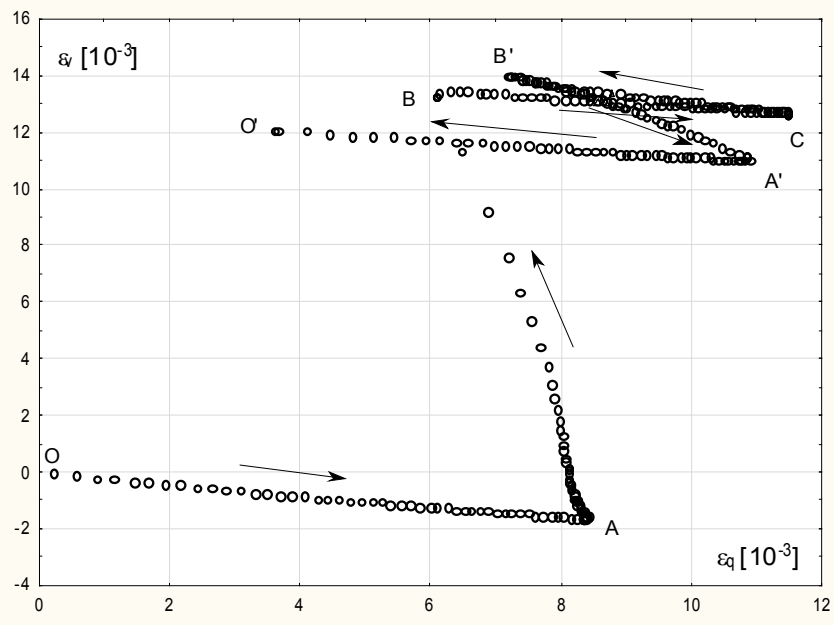

Fig. 5. The strain path corresponding to the stress path from Fig. 4 for an initially dilative soil 
dilative soil. The behavior of an initially contractive soil is similar from the qualitative point of view. The only essential difference is the positive slope of the sector $\mathrm{AB}$, as the compaction takes place during shearing.

Figure 5 is very interesting, as it shows almost linear relationships between volumetric and deviatoric strains along each sector of the stress path. The real experimental data are not entirely linear, but the linear approximation of these results is acceptable. Table 3 summarizes conclusions from the analysis of Fig. 5.

Table 3. Interpretation of strain increments corresponding to the stress path from Fig. 4 (initially dilative soil)

\begin{tabular}{|c|c|l|}
\hline Sector & $\begin{array}{c}\text { Strain } \\
\text { increments }\end{array}$ & \multicolumn{1}{c|}{ Comments } \\
\hline $0 \mathrm{~A}$ & $d \varepsilon_{v}>0, d \varepsilon_{q}<0$ & $\begin{array}{l}\text { Volumetric strain increases } \\
\text { (compaction); } \\
\text { Deviatoric strains develop due to } \\
\text { anisotropic character of the soil sample. }\end{array}$ \\
\hline $\mathrm{AB}$ & $d \varepsilon_{v}<0, d \varepsilon_{q}>0$ & $\begin{array}{l}\text { Volumetric strain decreases } \\
\text { (expansion in volume) due to dilation; } \\
\text { Deviatoric strain drastically increases. }\end{array}$ \\
\hline $\mathrm{BC}$ & $d \varepsilon_{v}>0, d \varepsilon_{q}<0$ & $\begin{array}{l}\text { Volumetric strain increases; } \\
\text { Deviatoric strain slightly decreases. }\end{array}$ \\
\hline $\mathrm{CB}$ & $d \varepsilon_{v}<0, d \varepsilon_{q}>0$ & $\begin{array}{l}\text { Volumetric strain decreases; } \\
\text { Deviatoric strain increases. }\end{array}$ \\
\hline $\mathrm{BA}$ & $d \varepsilon_{v}>0, d \varepsilon_{q}<0$ & $\begin{array}{l}\text { Volumetric strain increases; } \\
\text { Deviatoric strain decreases. }\end{array}$ \\
\hline $\mathrm{A} 0$ & $d \varepsilon_{v}<0, d \varepsilon_{q}>0$ & $\begin{array}{l}\text { Volumetric strain decreases; } \\
\text { Deviatoric strain increases. }\end{array}$ \\
\hline
\end{tabular}

The interpretation of experimental results along the sectors $0 \mathrm{~A}, \mathrm{AB}, \mathrm{BA}$ and $\mathrm{A} 0$ poses no problems, as we are dealing with unique processes that are consistent with physical intuition. For example, spherical loading takes place along the path $0 \mathrm{~A}$, and pure deviatoric loading takes place along the path $\mathrm{AB}$. Note that the signs of $d q$ and $d \eta$ are the same along the path $\mathrm{AB}$. A certain problem arises when we study the soil behavior along the paths $\mathrm{BC}$ and $\mathrm{CB}$, where the signs of $d q$ and $d \eta$ are different, see Table 2. There are a few ways to solve this dilemma. The simplest is to accept definitions introduced in Section 5 along with the consequences of such a choice. Another possibility is to link the processes of deviatoric loading/unloading with the corresponding increase/decrease in deviatoric strain. The comparison of Tables 2 and 3 shows that a consistent choice is to define $d \eta>0$ as deviatoric loading and $d \eta<0$ as deviatoric unloading. In the first case $d \varepsilon_{q}>0$, whereas in the second $d \varepsilon_{q}<0$, which agrees with physical intuition.

\section{SIMPLE CALIBRATION OF INCREMENTAL EQUATIONS}

A general method for the calibration of constitutive equations was described in Section 6. In this section, it will be shown how this method can be applied to calibrate the incremental equations (1) and (2). The starting point are the stress-strain relations shown in Figs. 6-9, corresponding to the stress path from Fig. 4. These charts deal with an initially dilative soil.

\section{Path 0A}

This path corresponds to spherical loading, during which both volumetric and deviatoric strains develop, see Figs. 6 and 8. They can be approximated by the following analytical formulae

$$
\begin{aligned}
& \varepsilon_{v}=\varepsilon_{v}^{\left(p^{\prime}\right)}=A_{v} \ln \left(p^{\prime}+1\right), \\
& \varepsilon_{q}=\varepsilon_{q}^{\left(p^{\prime}\right)}=A_{q} \ln \left(p^{\prime}+1\right),
\end{aligned}
$$

where $A_{v}$ and $A_{q}$ are coefficients. Their values in the case considered are the following $A_{v}=8.24$ and $A_{q}=$ -1.67 , for stress unit $10^{5} \mathrm{~N} / \mathrm{m}^{2}$ and strain unit $10^{-3}$. For example, if $p^{\prime}=200 \mathrm{kPa}=2 \times 10^{5} \mathrm{~N} / \mathrm{m}^{2}$, we obtain the following value of the deviatoric strain $\varepsilon_{q}=-1.72$ in unit $10^{-3}$. This means that the corresponding nondimensional strain is just $-1.72 \times 10^{-3}=-0.00172$. Recall that the form of analytical approximations is a matter of convenience, cf. equations (20)-(22) and the corresponding comments.

Differentiation of equations (28) and (29) with respect to $p^{\prime}$ leads to the following formulae:

$$
\begin{gathered}
\frac{\partial \varepsilon_{v}}{\partial p^{\prime}}=\frac{A_{v}}{1+p^{\prime}}=M^{\prime}=M^{l}, \\
\frac{\partial \varepsilon_{q}}{\partial p^{\prime}}=\frac{A_{q}}{1+p^{\prime}}=P^{\prime}=P^{l},
\end{gathered}
$$

see equations (3) and (4). The subscript, denotes loading.

\section{Path AB}

The path $\mathrm{AB}$ corresponds to deviatoric loading, and also along this path both deviatoric and volumetric strains develop, see Figs. 7 and 9. Experimentally obtained curves can be approximated by the following analytical formulae 


$$
\begin{gathered}
\varepsilon_{q}=\varepsilon_{q}^{(\eta)}=\frac{c \sqrt{p^{\prime}} \eta}{\eta^{\prime \prime}-\eta}+\text { const } 1 \\
\varepsilon_{v}=\varepsilon_{v}^{(\eta)}=\sqrt{p^{\prime}}\left(a_{1} \exp \left(a_{2} \eta\right)+a_{3} \eta\right)+\text { const } 2
\end{gathered}
$$

where $c, a_{1}, a_{2}, a_{3}$ are coefficients, and $\eta^{\prime \prime}$ corresponds to the Coulomb-Mohr yield condition. For an initially dense sand, characterized by $\varphi=41^{\circ}$, there is $\eta^{\prime \prime}=$ 1.68, see Sawicki and Świdziński [8]. The corresponding constants have no direct physical meaning, as they have only to assure the continuity of strains along a given stress path.

In the case considered, these coefficients take the following values: $c=0.96, a_{1}=-0.26 \times 10^{-5}, a_{2}=$ $8.45, a_{3}=-0.18$. The corresponding constants are the following: const $1=-1.72$, const $2=8.5$. Recall the units applied. Also note that during the experiment described $p^{\prime}=1.8$ in the assumed unit. Partial differentiation of equations (32)-(33) leads to the following formulae

$$
\begin{gathered}
\frac{\partial \varepsilon_{q}}{\partial \eta}=\frac{c \sqrt{p^{\prime}} \eta^{\prime \prime}}{\left(\eta^{\prime \prime}-\eta\right)^{2}}=Q^{\prime}=Q^{l}, \\
\frac{\partial \varepsilon_{v}}{\partial \eta}=\sqrt{p^{\prime}}\left(a_{1} a_{2} \exp \left(a_{2} \eta\right)+a_{3}\right)=N^{l} .
\end{gathered}
$$

\section{Path A0}

This path corresponds to spherical unloading. The corresponding experimental curves from Figs. 6 and 8 can be approximated by the following analytical formulae:

$$
\begin{gathered}
\varepsilon_{v}=a_{v} \ln \left(1+p^{\prime}\right)+\text { const } 3, \\
\varepsilon_{q}=b_{q} \sqrt{p^{\prime}}+\mathrm{const} 4,
\end{gathered}
$$

where $a_{v}=7.64, b_{q}=-0.805$, const $3=3.8$, const $4=12$. Differentiation of these formulae with respect to $p^{\prime}$ leads to the following equations

$$
\begin{aligned}
& \frac{\partial \varepsilon_{v}}{\partial p^{\prime}}=\frac{a_{v}}{1+p^{\prime}}=M^{u}, \\
& \frac{\partial \varepsilon_{q}}{\partial p^{\prime}}=\frac{b_{q}}{2 \sqrt{p^{\prime}}}=P^{u},
\end{aligned}
$$

where the subscript ()$^{u}$ denotes unloading.

\section{Path BA}

This path corresponds to deviatoric unloading. The corresponding curves from Figs. 7 and 9 can be approximated as follows

$$
\begin{aligned}
& \varepsilon_{v}=\sqrt{p^{\prime}}\left(b_{1} \eta^{2}+b_{2} \eta\right)+\text { const } 5, \\
& \varepsilon_{q}=\sqrt{p^{\prime}}\left(c_{1} \eta^{2}+c_{2} \eta\right)+\text { const } 6,
\end{aligned}
$$

where $b_{1}=0.775, b_{2}=-2.885, c_{1}=-1.21, c_{2}=3.15$, const $5=11$, const $6=11$. Differentiation of the above formulae with respect to $\eta$ leads to the following relations

$$
\begin{aligned}
& \frac{\partial \varepsilon_{v}}{\partial \eta}=\sqrt{p^{\prime}}\left(2 b_{1} \eta+b_{2}\right)=N^{u}, \\
& \frac{\partial \varepsilon_{q}}{\partial \eta}=\sqrt{p^{\prime}}\left(2 c_{1} \eta+c_{2}\right)=Q^{u} .
\end{aligned}
$$

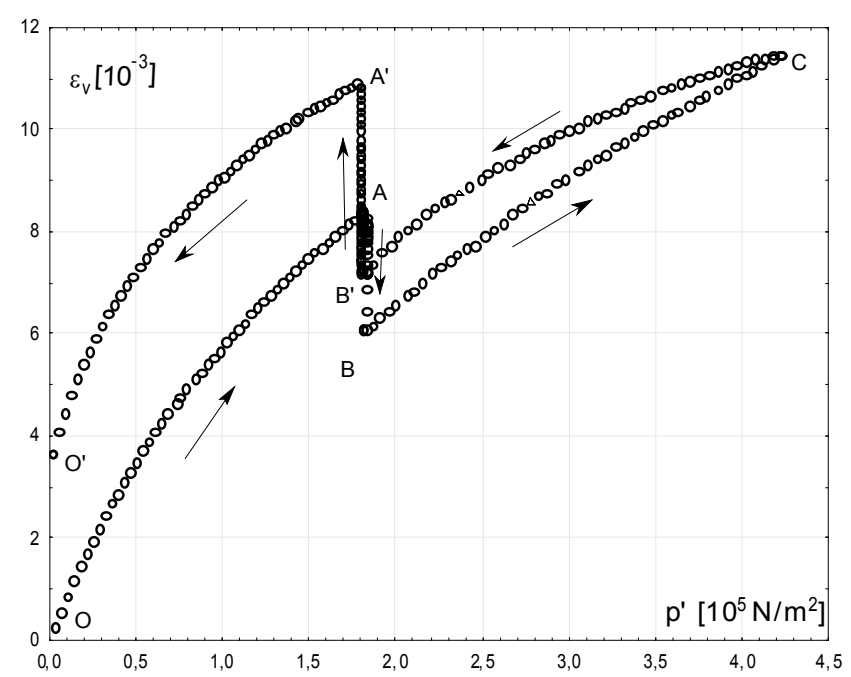

Fig. 6. Volumetric strain as a function of $p^{\prime}$ for the stress path shown in Fig. 4

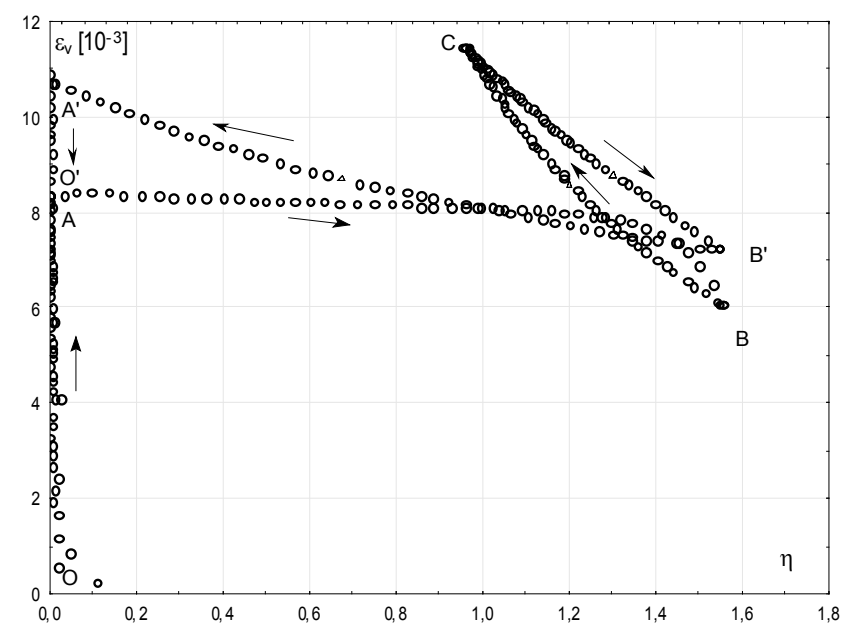

Fig. 7. Volumetric strain as a function of the non-dimensional stress $\eta$ for the stress path from Fig. 4 


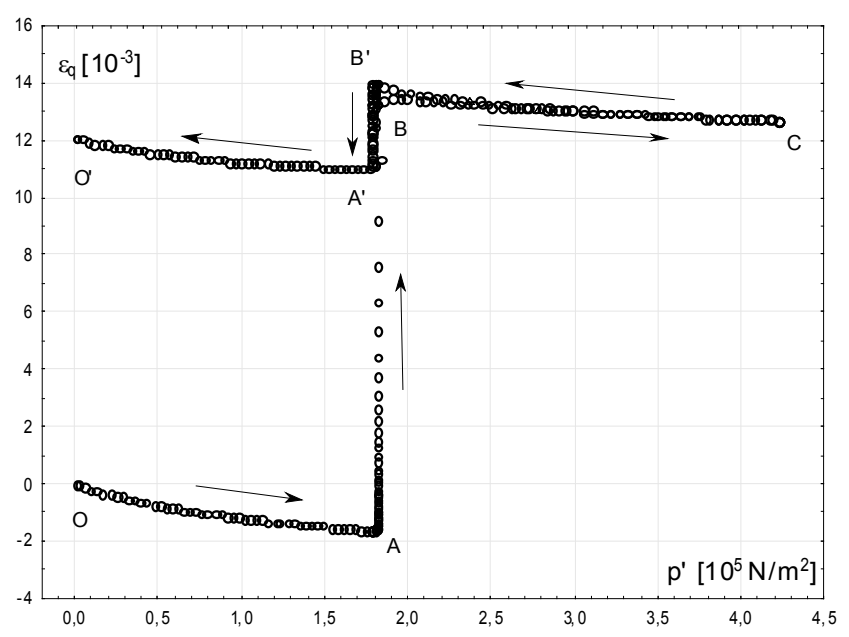

Fig. 8. Deviatoric strain as a function of $p^{\prime}$ for the stress path from Fig. 4

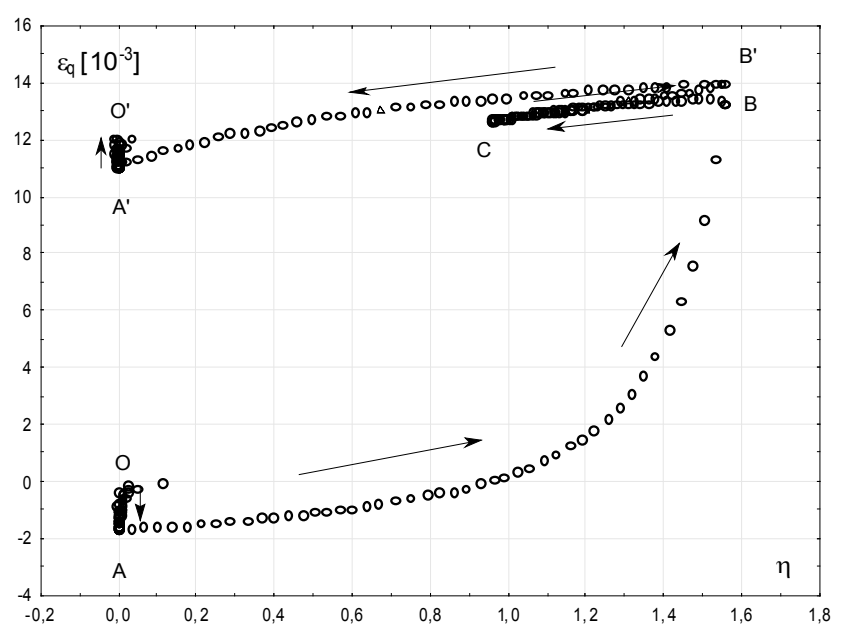

Fig. 9. Deviatoric strain as a function of $\eta$ for the stress path from Fig. 4

\section{PARTIAL VERIFICATION OF INCREMENTAL EQUATIONS}

The procedure described in Section 5 makes it possible to calibrate incremental equations on the basis of a single experiment. A more general approach to this problem, as well as to the problem of how to verify constitutive equations, is described in Sawicki and Świdziński [8], [9]. In this section, only a partial verification of constitutive functions will be presented for the sake of illustration. Recall that we have calibrated the model using the experimental stress-strain curves corresponding to the stress paths $\mathrm{OA}$ and $\mathrm{AB}$ (loading), and $\mathrm{A} 0$ and $\mathrm{BA}$ (unloading). Let us see the predictions of the model for the stress paths $\mathrm{BC}$ and $\mathrm{CB}$. The corresponding experimental results are shown in Figs. 6-9, and they will serve as reference curves for theoretical predictions.

\section{Path BC}

This path corresponds to spherical loading and deviatoric unloading, see Table 3. Therefore, the incremental equations (3) and (4) take the following form

$$
\begin{gathered}
d \varepsilon_{v}=M^{u} d p^{\prime}+N^{u} d \eta, \\
d \varepsilon_{q}=P^{l} d p^{\prime}+Q^{l} d \eta,
\end{gathered}
$$

where the corresponding constitutive functions are given by equations (30), (31), (42) and (43). The stress path $\mathrm{BC}$ is given by the following relation: $q=0.54 p^{\prime}+1.89$. Therefore,

$$
\begin{gathered}
\eta=q / p^{\prime}=0.54+1.89 / p^{\prime} \\
\text { and } \quad d \eta=-\frac{1.89}{\left(p^{\prime}\right)^{2}} d p^{\prime} .
\end{gathered}
$$

After substitution of the corresponding coefficients and equation (46) into the relations (44) and (45), one obtains:

$$
d \varepsilon_{v}=\left(\frac{A_{v}}{1+p^{\prime}}-\frac{1.89}{p^{\prime 2}} \sqrt{p^{\prime}}\left(2 b_{1} \frac{0.54 p^{\prime}+1.89}{p^{\prime}}+b_{2}\right)\right) d p^{\prime},
$$

$$
d \varepsilon_{q}=\left(\frac{A_{q}}{1+p^{\prime}}-\frac{1.89}{p^{\prime 2}} \sqrt{p^{\prime}}\left(2 c_{1} \frac{0.54 p^{\prime}+1.89}{p^{\prime}}+c_{2}\right)\right) d p^{\prime} \text {. }
$$

Integration of the above equations leads to the following formulae for the corresponding strains

$$
\begin{gathered}
\varepsilon_{v}^{B C}=A_{v} \ln \left(1+p^{\prime}\right)+4.762 b_{1}\left(p^{\prime}\right)^{-\frac{1}{2}} \\
+4.763 b_{1}\left(p^{\prime}\right)^{-\frac{3}{2}}+3.78 b_{2}\left(p^{\prime}\right)^{-\frac{1}{2}}+1.25, \\
\varepsilon_{q}^{B C}=A_{q} \ln \left(1+p^{\prime}\right)+4.762 c_{1}\left(p^{\prime}\right)^{-\frac{1}{2}} \\
+4.763 c_{1}\left(p^{\prime}\right)^{-\frac{3}{2}}+3.78 c_{2}\left(p^{\prime}\right)^{-\frac{1}{2}}+13 .
\end{gathered}
$$

Figure 10 shows measured (cf. Figs. 6 and 8) and predicted (equations 48 and 49) stress-strain curves. The agreement is very good. 


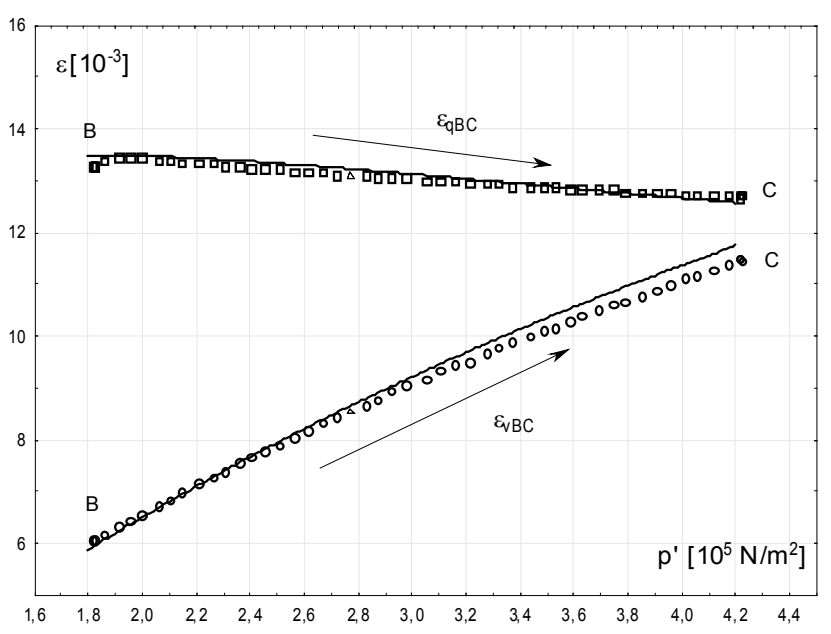

Fig. 10. Measured and predicted strain-stress curves

\section{Path CB'}

Volumetric and deviatoric strain increments are as follows

$$
\begin{aligned}
& d \varepsilon_{v}=M^{u} d p^{\prime}+N^{u} d \eta, \\
& d \varepsilon_{q}=P^{u} d p^{\prime}+Q^{u} d \eta .
\end{aligned}
$$

The stress path $\mathrm{CB}^{\prime}$ can be expressed as: $q=0.54 p^{\prime}$ +1.89 , therefore $d \eta=-\frac{1.89}{\left(p^{\prime}\right)^{2}} d p^{\prime}$.

Substitution of equations (38) and (42) into equation (51) gives

$$
d \varepsilon_{v}=\left(\frac{a_{v}}{1+p^{\prime}}-\frac{1.89}{p^{\prime 2}} \sqrt{p^{\prime}}\left(2 b_{1} \frac{0.54 p^{\prime}+1.89}{p^{\prime}}+b_{2}\right)\right) d p^{\prime} .
$$

Integration of equation (53) gives

$$
\begin{aligned}
& \varepsilon_{v}^{C B}=a_{v} \ln \left(1+p^{\prime}\right)-a_{v} \ln (5.2)+4.0824 b_{1} p^{\prime-\frac{1}{2}} \\
& +4.7628 b_{1} p^{\prime-\frac{3}{2}}+3.78 b_{2} p^{-\frac{1}{2}}-4.0824 b_{1}(4.2)^{-\frac{1}{2}} \\
& -4.7628 b_{1}(4.2)^{-\frac{3}{2}}-3.78 b_{2}(4.2)^{-\frac{1}{2}}+11.75 .
\end{aligned}
$$

Substitution of equations (39) and (43) into equation (52) gives

$$
d \varepsilon_{q}^{C B}=\left(\frac{b_{q}}{2 \sqrt{p^{\prime}}}-\frac{1.89}{p^{\prime 2}} \sqrt{p^{\prime}}\left(2 c_{1} \frac{0.54 p^{\prime}+1.89}{p^{\prime}}+c_{2}\right)\right) d p^{\prime} .
$$

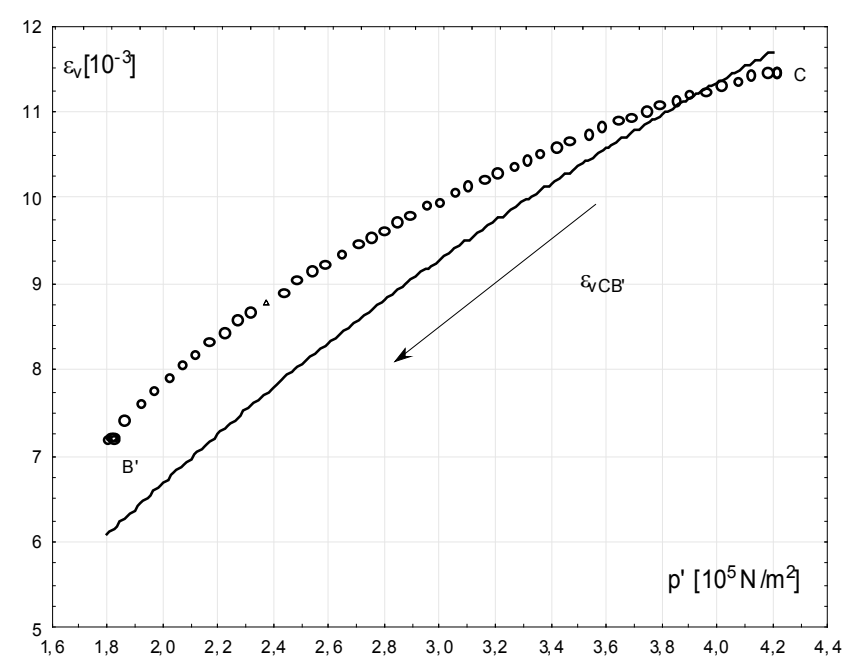

Fig. 11. Volumetric strains caused by the stress path $\mathrm{CB}^{\prime}$

Integration of equation (55) leads to the following formula

$$
\begin{aligned}
\varepsilon_{q}^{C B}= & b_{q} \sqrt{p^{\prime}}-b_{q} \sqrt{4.2}+4.0824 c_{1} p^{-\frac{1}{2}}+4.7628 c_{1} p^{-\frac{3}{2}} \\
& +3.78 c_{2} p^{-\frac{1}{2}}-4.0824 c_{1}(4.2)^{-\frac{1}{2}} \\
& -4.7628 c_{1}(4.2)^{-\frac{3}{2}}-3.78 c_{2}(4.2)^{-\frac{1}{2}}+12.7 .
\end{aligned}
$$

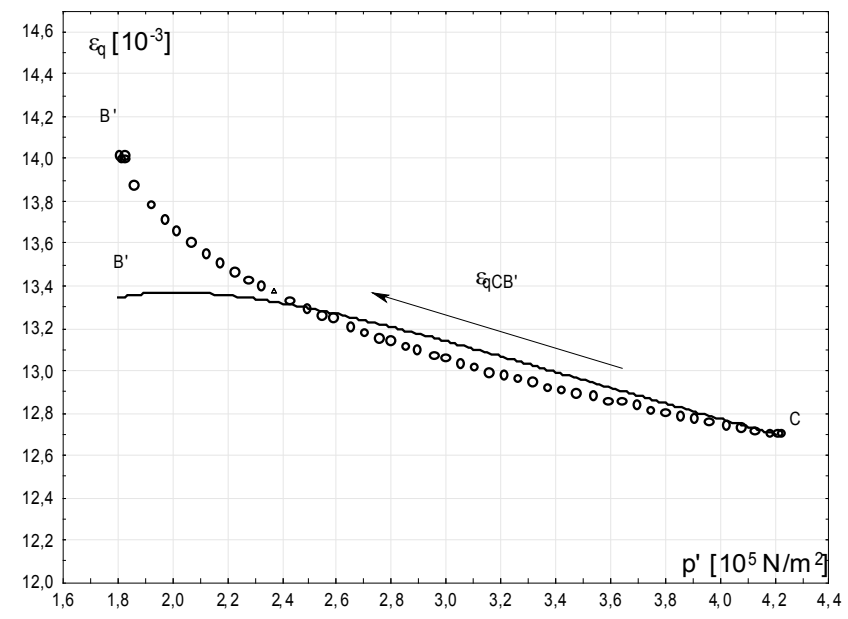

Fig. 12. Deviatoric strains caused by the stress path $\mathrm{CB}^{\prime}$

The strains given by equations (54) and (55) are illustrated in Figs. 11 and 12. The volumetric strains are predicted with a reasonable accuracy, whereas the deviatoric strain is comfortable with experimental data from $4.2 \times 10^{5} \mathrm{~N} / \mathrm{m}^{2}$ down to $2.4 \times 10^{5} \mathrm{~N} / \mathrm{m}^{2}$. For smaller values of the mean effective stress, however, both curves diverge for an unknown reason. Recall that a single experiment is analysed. 
Table 4. Comparison between the classical elasto-plastic approach and the semi-empirical model of the pre-failure behaviour of granular soils

\begin{tabular}{|c|c|c|}
\hline Definition & Classical elasto-plasticity & Semi-empirical model \\
\hline 1. Limit/critical state & Coulomb-Mohr criterion & Coulomb-Mohr criterion \\
\hline 2. Yield condition/surface & $\begin{array}{l}\text { Yield surfaces in statically admissible region } \\
\text { introduced. They may develop during loading, } \\
\text { depending on the hardening rule. In an ideal } \\
\text { elasto-plastic approach, the behaviour of soil } \\
\text { inside the limit surface is assumed as elastic. }\end{array}$ & Yield surfaces are not introduced. \\
\hline 3. Flow rule & $\begin{array}{l}\text { A flow rule is assumed in order to determine } \\
\text { plastic strain increments. These rule is assumed } \\
\text { either as associated or non-associated. In the } \\
\text { latter case, a new function (plastic potential) } \\
\text { is introduced. }\end{array}$ & Flow rules are not introduced. \\
\hline 4. Loading/unloading & $\begin{array}{l}\text { Subsequent stress increments denote loading or } \\
\text { unloading depending on their orientation with } \\
\text { respect to the current yield surface. }\end{array}$ & $\begin{array}{l}\text { A natural definition of loading/ } \\
\text { unloading is introduced. The distinction } \\
\text { is made between spherical and deviatoric } \\
\text { loading/unloading. }\end{array}$ \\
\hline 5. Strains & $\begin{array}{l}\text { Plastic strains calculated by the integration of } \\
\text { incremental equations, obtained from the flow } \\
\text { rule, for a given loading path. Elastic strains } \\
\text { are usually calculated from Hooke's law. }\end{array}$ & The model is formulated for total strains. \\
\hline 6. Initial state of soil & $\begin{array}{l}\text { Usually, these models do not take into account } \\
\text { the initial state, defined either as contractive } \\
\text { or dilative. }\end{array}$ & $\begin{array}{l}\text { The initial state (contractive or dilative) } \\
\text { is taken into account. }\end{array}$ \\
\hline 7. Instability line & Usually not introduced. & $\begin{array}{l}\text { Taken into account. } \\
\text { See Sawicki and Świdziński [8], [9]. }\end{array}$ \\
\hline 8. Anisotropy of soil & Usually ignored. & Taken into account. \\
\hline
\end{tabular}

\section{CONCLUSIONS}

The main result of this paper is to show a potential of semi-empirical piece-wise linear incremental equations for the description of the pre-failure behaviour of granular soils. The structure of these equations was presented, and the method of calibration and partial verification described. This structure was described in the context of the classical elasto-plastic approach. A summary of these approaches is presented in Table 4.

\section{REFERENCES}

[1] AtKInson J.H., An Introduction to the Mechanics of Soil and Foundations, McGraw-Hill, London 1993.

[2] BAZANT Z.P., KRIZEK R.J., Endochronic constitutive law for liquefaction with cyclic loads, Proc. ASCE, J. Engrg. Mech. Div., 1976, 102, EM2, 225-238.
[3] GudEHUS G., A comprehensive constitutive equation for granular materials, Soils and Foundations, 1996, 36, No. 1, 1-12.

[4] Kolymbas D., Introduction to Hypoplasticity, Advances in Geotechnical Engineering and Tunnelling, Balkema, Rotterdam/Brookfield 2000.

[5] SaAda A., Bianchini G. (eds.), Constitutive equations for granular non-cohesive soils, Proc. Int. Workshop, Cleveland, Balkema, Rotterdam/Brookfield 1987.

[6] SAwICKI A., Zarys mechaniki gruntów sypkich, IBW PAN, Gdańsk 2012.

[7] SAWICKI A., The puzzle of liquefaction, IBW PAN, Gdańsk 2014.

[8] SAWICKI A., ŚWIDZIŃSKI W., Stress-strain relations for dry and saturated sands, Part I: Incremental model, J. Theoretical and Applied Mechanics, 2010a, 48, 2, 309-328.

[9] SAWICKI A., ŚWIDZIŃSKI W., Stress-strain relations for dry and saturated sands, Part II: Predictions, J. Theoretical and Applied Mechanics, 2010b, 48, 2, 329-373.

[10] VALANIS K.C., An Endochronic Geomechanical Model For Soils, [in:] Deformation and Failure of Granular Materials, (ed. P.A. Vermeer, H.J. Luger), A. A. Balkema, Rotterdam 1082, 159-165.

[11] Wood D.M., Soil Behaviour and Critical State Soil Mechanics, Cambridge University Press, Cambridge 1990. 